\title{
Une mutation du gène du récepteur du glucagon dans le diabète non insulinodépendant
}

Le diabète non insulinodépendant (DNID) est une affection fréquente, (touchant environ $5 \%$ de la population mondiale), cliniquement et génétiquement très hétérogène. Les études de jumeaux ont montré l'importance des facteurs génétiques dans sa transmission, même s'il paraît polygénique dans la plupart des cas. De nombreux gènes candidats ont été étudiés, conduisant à l'identification de mutations dans des groupes particuliers de patients diabétiques : mutations des gènes de la glucokinase dans le DNID à début précoce ou MODY (maturity onset diabetes of the young), du récepteur de l'insuline dans le lépréchaunisme, de l'insuline, mutation de l'ADN mitochondrial dans le DNID à transmission maternelle... Cependant, toutes ces mutations expliquent probablement moins de $5 \%$ de l'ensemble des diabètes [1] et, jusqu'à ce jour, aucune mutation n'avait été associée aux formes communes de DNID à début tardif.

Le glucagon, produit par les cellules $\alpha$ des îlots de Langherans pancréatiques, est, avec l'insuline, une des deux hormones clés de l'homéostasie glycémique. Son action est essentiellement hyperglycémiante. Elle contrôle, en particulier, la production hépatique de glucose, réglant la néoglucogenèse, et surtout la glycogénolyse. Cependant, le glucagon intervient aussi dans la régulation de l'insulinosécrétion, avec d'autres hormones digestives comme le GIP* et surtout le GLP $1^{* *}$, pour amplifier la réponse au glucose ou aux acides aminés.

Les concentrations de glucagon chez les patients DNID sont, paradoxale-

* GIP: gastric inhibitory peptide.

** GIP : glucagon-like peptide, fragment $1 d u$ ment, souvent élevées, malgré leur hyperglycémie, ce qui contribue probablement à aggraver le diabète par l'effet hépatique du glucagon. Le mécanisme de l'hyperglucagonémie est inconnu, et l'on évoque une "cécité " des cellules $\alpha$ au rétrocontrôle par la glycémie. Une autre explication résiderait dans une anomalie primitive de la réponse au glucagon lui-même. Le glucagon agit par l'intermédiaire d'un récepteur membranaire qui fait partie de la superfamille des récepteurs transmembranaires couplés à des protéines $\mathrm{G}\left(\mathrm{m} / \mathrm{s} n^{\circ} 4\right.$, vol. 9, p. 472). Une diminution de l'activité du récepteur du glucagon pourrait perturber les propriétés insulinotropes du glucagon, avec hyperproduction compensatrice de l'hormone par les cellules $\alpha$ des îlots de Langherans. Les gènes de rat et humain du récepteur du glucagon ont été récemment clonés. Chez l'homme, le gène comporte 13 exons, et s'étend sur $5,5 \mathrm{~kb}$ sur le chromosome $17 q 25$. Il a été possible de rechercher des mutations de ce gène par l'utilisation de la technique de SSCP-PCR (single strand conformational polymorphism) [2] chez des patients DNID.

Ces travaux viennent de conduire à l'identification d'une mutation unique, située sur le codon 40 de l'exon 2, qui transforme un triplet GGT (Gly) en AGT (Ser) (mutation G40S). Il est à noter que ce résidu Ser constitue la seule différence entre les séquences humaine et murine, dans une zone de 49 acides aminés identiques entre les deux espèces. La mutation G40S est présente chez $5 \%$ des diabétiques français testés et chez $8 \%$ d'une population de patients sardes [3]. En revanche, la mutation G40S était totalement absente d'une population de sujets non diabétiques et sans antécédents familiaux de DNID, et est très rare (moins de $1 \%$ ) dans la population générale. Il existe donc une association entre le DNID à début tardif et le gène du récepteur du glucagon. L'étape suivante était de rechercher une liaison génétique entre la maladie et ce marqueur dans des familles diabétiques dont certains membres sont porteurs de la mutation. Cette liaison était difficile à tester par la méthode des lod scores, compte tenu du caractère polygénique du mode de transmission du DNID. De plus, le pouvoir de l'analyse de sib-pairs* est trop faible dans le cadre d'un variant rare, à proximité duquel aucun polymorphisme fortement informatif n'a été décrit (le gène du récepteur du glucagon est en effet très télomérique). C'est pourquoi, l'analyse de choix est la recherche d'un déséquilibre de transmission du variant de parents hétérozygotes à des enfants affectés (transmission disequilibrium test, TDT). Cette méthode a été utilisée avec succès pour mettre en évidence le rôle du gène de l'insuline dans le diabète insulinodépendant (dans ce cas, il s'agissait, au contraire, d'un haplotype de susceptibilité très fréquent, dont la pénétrance était très faible, [4]). La fréquence de transmission de la mutation G40S de parents hétérozygotes aux enfants affectés a été de $73 \%$, soit significativement plus que les $50 \%$ attendus en cas d'hypothèse nulle (absence de linkage). La liaison génétique était aussi significative si l'on ne prenait en compte que les sujets les moins atteints, présentant une simple intolérance au gluco-

* Recherche d'un excès de concordance (>50 \%)
dans la transmission génique des haplotypes à proxi-
mité d'un gène morbide parmi les paires de germains affectés étudiés. 
se, ce qui suggère que la mutation du gène du récepteur du glucagon joue un rôle dès les premiers stades de la maladie. Il restait, bien entendu, à étudier les effets biologiques de cette mutation dans des cellules eucaryotes (cellules de rein de hamster, BHK). Après introduction de la mutation dans l'ADNc du récepteur du glucagon par mutagenèse dirigée, l'analyse des propriétés de liaison du récepteur a montré que l'affinité pour le glucagon était divisée par trois, par rapport au type original. Selon des données encore préliminaires, il semble que la production d'AMPc soit aussi diminuée dans ces cellules, ainsi que dans des cellules insulinosécrétrices en culture (cellules RIN) issues d'un insulinome de rat.

Il reste à comprendre l'implication du gène du récepteur du glucagon dans la physiopathologie du DNID. L'hypothèse la plus probable, étant donnée la diminution de l'activité fonctionnelle du variant, fait intervenir un trouble de l'insulino-sécré- tion. Les patients porteurs de la mutation G40S n'ont pas de caractéristique clinique très particulière, en dehors d'un poids plus faible que les diabétiques sans cette mutation. Des études métaboliques chez les diabétiques porteurs de la mutation, en particulier la réponse pancréatique à l'injection de glucagon, la sécrétion de glucagon après stimulation par l'hypoglycémie ou des acides aminés et la production hépatique du glucose permettront de mieux comprendre les troubles en cause. La prévalence de la mutation du gène du récepteur du glucagon reste à déterminer dans d'autres populations. Selon des résultats encore préliminaires, environ $2 \%$ de patients britanniques non sélectionnés auraient cette même mutation, qui serait extrêmement rare $(0,2 \%)$ chez des sujets non diabétiques (John Todd, résultats non publiés). L'association entre le gène du récepteur du glucagon et le DNID semble confirmée au Danemark et en Allemagne.
En conclusion, quoique le gène du récepteur du glucagon ne soit qu'un des déterminants génétiques de la forme polygénique du DNID, son identification constitue, néanmoins, un pas de plus dans le démembrement nosologique de ce syndrome hétérogène.

P.F.

1. Froguel P, Vionnet N, Gauguier D, Vaxillaire M, Zouali H, Passa P, Velho G. Génétique du diabète non insulinodépendant. médecine/sciences $1994 ; 10$ : 795-804.

2. Dreyfus JC, Akli S, Poenaru L. Maladies de Tay Sachs et de Sandhoff . Les déficits en $\beta$-hexosaminidases, modèles des maladies des lysosomes. $m \dot{e}$ decine/sciences $1992 ; 8 \quad 797-803$.

3. Hager J, Hansen L, Vaisse C, Vionnet N, Philippi A, Poller W, Velho G, Carcassi C, Contu L, Julier J, Cambien F, Passa P, Lathrop M, Kindsvogel W, Demenais F, Nishimura E, Froguel P. A missense mutation in the glucagon receptor gene is associated with familial non-insulino-dependent diabetes mellitus. Nature Genet 1995 (sous presse). 4. Froguel $P$. Un nouveau gène $d$ u diabète insulino-dépendant sur le chromosome llq. Le DID, paradigme pour l'étude des maladies multifactorielles. médecine/sciences $1994 ; 10: 1147-9$. 